\title{
Case Report \\ Use of Extracorporeal Membrane Oxygenation in Pneumocystis Pneumonia of an Infant with AIDS
}

\author{
Grégoire Cane $\mathbb{D}^{1,2}$ Arnaud De Boislambert, ${ }^{1}$ Charlotte Sgro, ${ }^{1}$ Pierre Lavedan, ${ }^{1}$ \\ Hélène Foulgoc, ${ }^{1}$ Nadir Tafer, ${ }^{1}$ and Alexandre Ouattara $\mathbb{D}^{1,2}$ \\ ${ }^{1}$ CHU Bordeaux, Department of Anaesthesia and Critical Care, Magellan Medico-Surgical Centre, F-33000 Bordeaux, France \\ ${ }^{2}$ Univ. Bordeaux, INSERM, UMR 1034, Biology of Cardiovascular Diseases, F-33600 Pessac, France \\ Correspondence should be addressed to Grégoire Cane; gregoire.cane@gmail.com
}

Received 26 August 2020; Accepted 9 November 2020; Published 18 November 2020

Academic Editor: Maria Moschovi

Copyright ( 92020 Grégoire Cane et al. This is an open access article distributed under the Creative Commons Attribution License, which permits unrestricted use, distribution, and reproduction in any medium, provided the original work is properly cited.

Pneumocystis pneumonia is a common complication of cellular immunosuppression and may trigger severe pulmonary complications. Rapid onset of acquired immunodeficiency syndrome is possible in infants infected with human immunodeficiency virus (HIV). We report here the case of a 13-week-old girl who was previously healthy presenting with altered immunity and refractory acute respiratory distress syndrome (ARDS) initially attributed to bacterial pneumonia. Venovenous extracorporeal membrane oxygenation (VV-ECMO) was initiated because her condition was poor. An HIV infection was later fortuitously diagnosed after accidental exposure of a nurse to the child's urine. The mother had congenitally transmitted HIV to the child after late (undetected) infection during pregnancy. The lung lesions were finally attributed to Pneumocystis pneumonia. We prescribed combined antiretroviral, antibiotic, and steroid therapy aimed at preventing immune reconstitution inflammatory syndrome. VV-ECMO weaning progressed over 30 days to the time of decannulation, rapidly followed by extubation and hospital discharge. The case highlights the fact that rare curable causes of refractory pediatric ARDS should always be investigated early. VV-ECMO should not be excluded as an ARDS treatment for immunocompromised children.

\section{Introduction}

Pneumocystis pneumonia is a common opportunistic infection in patients infected with human immunodeficiency virus (HIV) and can cause acute respiratory distress syndrome (ARDS) associated with a high mortality rate (up to $58 \%$ even in intensive care units (ICUs)) [1]. Venovenous extracorporeal membrane oxygenation (VV-ECMO) for adults with acquired immunodeficiency syndrome (AIDS) is generally associated with favorable outcomes [2-5]. However, no pediatric case has been described; the utility of VVECMO in children is questionable. Pediatric HIV infection can rapidly evolve to AIDS before 2 years of age; Pneumocystis pneumonia is not rare in patients with vertically transmitted HIV [6,7]. We present here the case of a congenitally HIV-infected infant who developed ARDS associated with refractory hypoxemia and Pneumocystis pneumonia. VV-ECMO, combined with cotrimoxazole and a corticosteroid, allowed complete respiratory function recovery. The HIV infection was fortuitously diagnosed, suggesting that immunosuppression status had not been adequately screened.

\section{The Case}

At the age of 13 weeks, a girl born at term who had previously been healthy was brought to the emergency unit of a local hospital in respiratory distress (day 0, see Figure 1 for the detailed timeline). The pregnancy had been uneventful; the 6-month maternal HIV screen was negative. The initial clinical evolution was pejorative; the child was placed under mechanical ventilation after orotracheal intubation on day 3 . At that time, diffuse alveolo-interstitial lesions were evident on a chest X-ray. Viral PCR revealed a rhinovirus in pulmonary secretions. No other infective agent was noted, but empirical antibiotics were commenced given the gravity of 


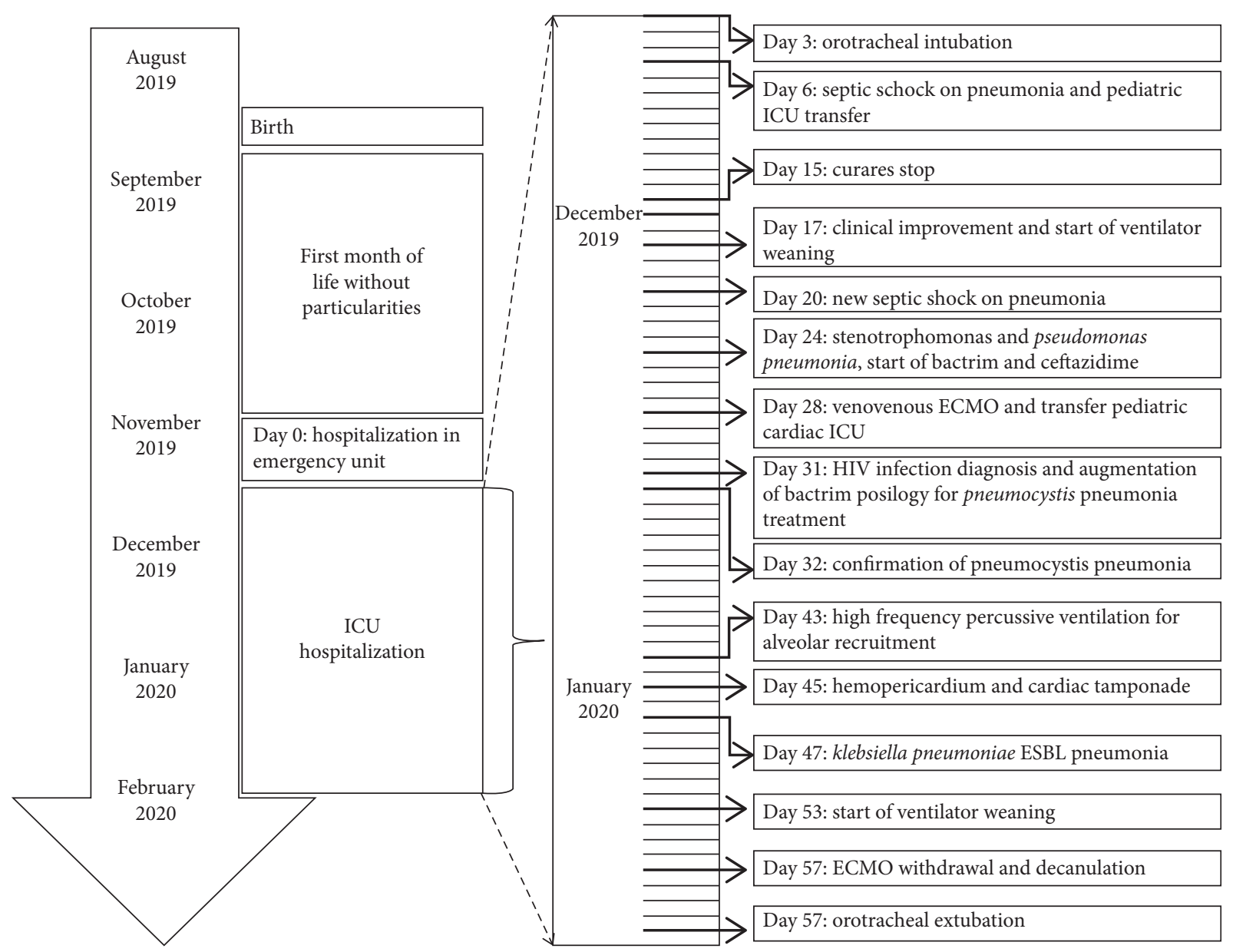

Figure 1: The patient's timeline.

the situation. The condition worsened during a flight between two ICU units, triggering an emergency landing. At arrival in a local, general pediatric ICU, curarization, optimization of ventilation, and prone positioning rapidly improved oxygenation. Such aggressive treatment encouraged the commencement of ventilator weaning on day 17 . The favorable clinical course ended on day 20; septic shock developed on ventilation, associated with pneumonia and bacteremia (Pseudomonas aeruginosa and Stenotrophomonas maltophilia). The antibiotics were changed to ceftazidime and cotrimoxazole. Given the new bacterial infections, possible immunosuppression was evaluated in terms of immunoglobulin and complement component levels; no abnormality was evident. Despite treatment reescalation and a priori antimicrobial therapy, her respiratory status worsened, and uncontrolled hypoxemia developed on day 28. No ventricular dysfunction was evident on transthoracic echocardiography, but major confluent bilateral opacities were apparent on a chest X-ray (Figure 2). Refractory ARDS in the absence of multivisceral failure or right ventricular dysfunction was attributed to bacterial pneumonia, justifying the use of VV-ECMO. After cannulation, the patient was transferred to the cardiac pediatric ICU. VVECMO afforded efficient gaseous exchange. Pulmonary compliance was very low at ICU arrival $\left(0.45 \mathrm{~mL} / \mathrm{cm} \mathrm{H}_{2} \mathrm{O}\right.$ with a tidal volume of $2 \mathrm{~mL} / \mathrm{kg}$ (i.e., $10 \mathrm{~mL}$ ); $8 \mathrm{~cm} \mathrm{H} \mathrm{H}_{2} \mathrm{O}$ of positive end-expiratory pressure (PEEP); and $22 \mathrm{~cm} \mathrm{H}_{2} \mathrm{O}$ of driving pressure). Antibiotic therapy (cotrimoxazole with $60 \mathrm{mg} / \mathrm{kg}$ sulfamethoxazole and $12 \mathrm{mg} / \mathrm{kg}$ trimethoprim) and ceftazidime were maintained.

A nurse was accidentally exposed to the child's urine (by pricking herself during collection). In order to decide if a postexposure prophylaxis was indicated, systematic evaluation of viral status of the patient was realized and revealed an HIV-positive serology; the child was found to be HIVpositive on day 31 . The viral load as revealed by RT-PCR was extremely high at $6.96 \quad \log _{10}$ copies $/ \mathrm{mL}$. The CD4+ lymphocyte count was very low at $72 / \mathrm{mm}^{3}$. The mother was diagnosed with an HIV infection that had developed late in pregnancy (after the 6-month screen), which had then been congenitally transmitted to the child. We screened for all classic AIDS co-opportunistic infections; all tests were negative except that for Pneumocystis carinii, which was strongly positive on PCR of a tracheal aspirate $(8,229$ copies $/ \mathrm{mL})$. On day 32 , the cotrimoxazole dose was changed to $100 \mathrm{mg} / \mathrm{kg} /$ day sulfamethoxazole combined with $20 \mathrm{mg} / \mathrm{kg} /$ day trimethoprim. Antiretroviral therapy was commenced in association with a corticosteroid $(1 \mathrm{mg} / \mathrm{kg} /$ day methylprednisolone) to prevent the development of immune reconstitution inflammatory syndrome. Such 


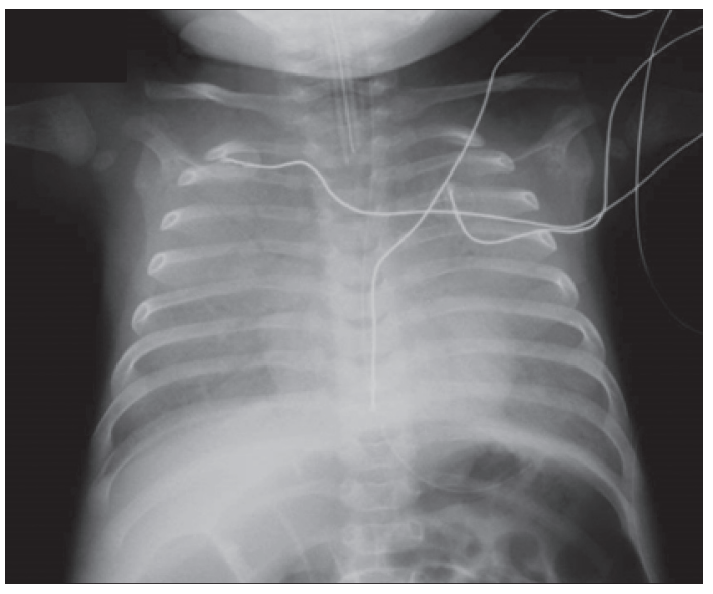

(a)

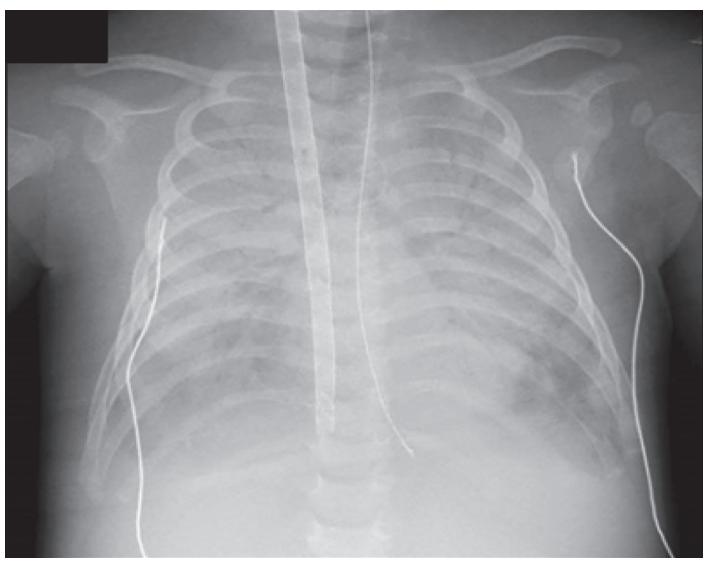

(c)

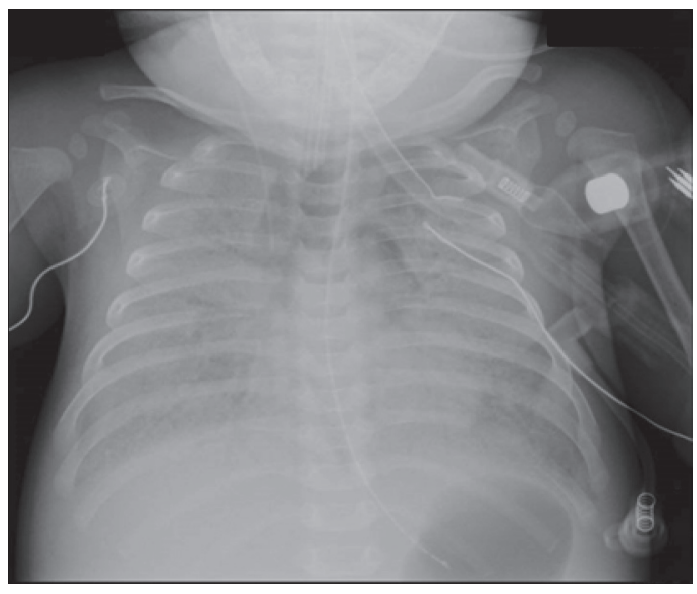

(b)

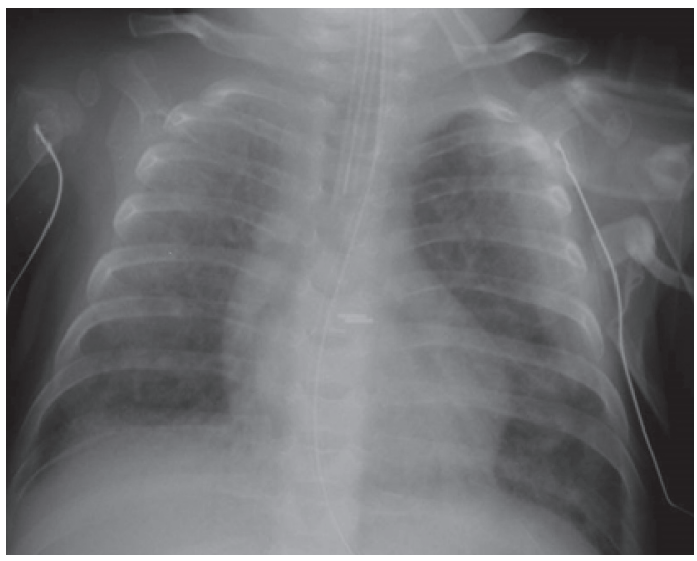

(d)

FIGURE 2: Chest X-rays taken on admission (a): on the day before cannulation and VV-ECMO commencement (b), on the day of VV-ECMO commencement (c), and on the day before extubation (d). Note the Avalon Cannula projecting in front of the superior and inferior vena cava in (c).

treatment greatly increased the daily fluid intake (the daily cotrimoxazole alone was delivered in $160 \mathrm{~mL}$ of solvent) of the $5 \mathrm{~kg}$ infant; we thus commenced VV-ECMO ultrafiltration to control the fluid balance.

Compliance slowly improved; computed tomography (Figure 3) revealed persistent pulmonary consolidation but without major fibrosis. More aggressive PEEP combined with sessions of percussive ventilation allowed alveolar lung recruitment. At day 45, the hemodynamic status became aggravated. Erosion of the right atrium caused by cannular malpositioning (amplified by the percussive ventilation) had created hemopericardium causing a cardiac tamponade. This was surgically treated; three interventions were required on the same day to iteratively evacuate hemoperitoneum emanating from a lesion in an abdominal arteriole created during the initial procedure. Despite this episode and a new bacterial pneumonia (caused by Klebsiella pneumoniae ESBL), her respiratory state progressively improved. Ventilator weaning commenced on day 53 with decannulation on day 57 and extubation and ICU discharge on day 61 . The patient was transferred a few days later to a dedicated AIDS center without any need for assisted ventilation. The infant's parents kindly gave us written informed consent for publication of this case report.

\section{Discussion}

To the best of our knowledge, this is the first report of successful ECMO treatment of refractory ARDS associated with Pneumocystis pneumonia in an AIDS infant. The Berlin criteria grade ARDS severity with reference to the $\mathrm{PaO}_{2}$ : $\mathrm{FiO}_{2}$ ratio; severe ARDS is associated with a value less than 100 [8]. Refractory ARDS is a form of severe ARDS that does not respond to mechanical ventilation, prone positioning, or muscle paralysis (to maintain blood saturation) [9]. VVECMO is the only method that prevents lung scarring and allows clinical recovery. The Extracorporeal Life Support Organization (ELSO) guidelines for pediatric respiratory failure [10] include a comprehensive list of scenarios in which patients benefit from such exceptional treatment. However, sometimes, ECMO applicability remains unclear. The contraindications [10] include a duration of pre-ECMO mechanical ventilation $>14$ days, a pre-existing chronic illness, and a poor long-term prognosis. Such conditions are 




(a)

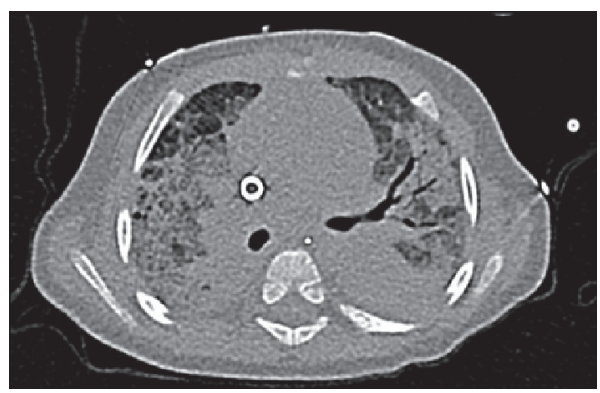

(c)



(b)

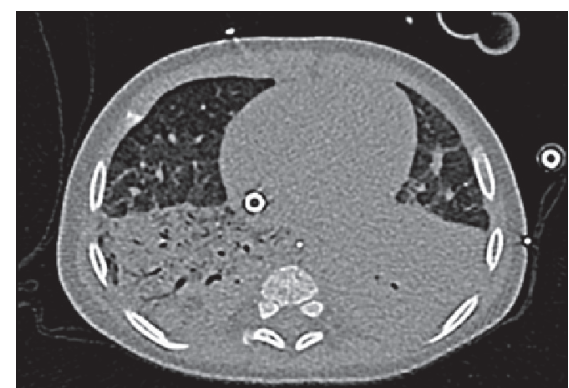

(d)

Figure 3: A thoracic computed tomograph taken during VV-ECMO-assisted recovery from Pneumocystis pneumonia-caused ARDS. (a) Apical lesions; (b) Carinal lesions associated with fibrosis and alveolar condensation; (c) lower lobe condensation (an air bronchogram); (d) lung base condensations. Note the Avalon Cannula in the vena cava in all panels.

associated with poorer hospital survival of pediatric patients recorded in large ELSO registries $[11,12]$. In our case, preECMO mechanical ventilation had been in place for 26 days, and an HIV infection (undiagnosed at the time of cannulation) was present. However, successful decannulation was possible 30 days later. Immunocompromised pediatric patients are at especially high risk of hospital death and are very susceptible to opportunistic infections [12] (survival rates of 33.3 and $57.5 \%$ for immunocompromised and nonimmunocompromised infants). However, some pediatric case reports of VV-ECMO used to treat Pneumocystis pneumonia reported that patients with hematological diseases and iatrogenic immunosuppression experienced good outcomes [13-15]. Also, the use of ECMO to treat Pneumocystis pneumonia was associated with better patient survival than that of patients with other conditions recorded in both the ELSO and Stockholm registries (51 and 89\% survival, respectively [16]). The curability of the underlying disease should be considered when deciding whether to commence ECMO. Immunosuppression caused by HIV infection should not prevent the use of VV-ECMO.

Another point of interest is the fortuitous diagnosis of an HIV infection after a nurse had been accidentally exposed to the child's urine. Prior to this discovery, apart from the bacteremia, we lacked clinical, biological, or radiological findings suggestive of immunocompromise. The diffuse alveolo-interstitial lung lesions and the severity of hypooxygenation could have been attributable to the initial rhinovirus infection associated with repeated bacterial infections. This case highlights the need to investigate rare but curable causes of ARDS systematically. Inappropriately, neither the ELSO [10] nor the Pediatric Acute Lung Injury Consensus Conference (PALICC) [9] recommended such investigations for pediatric patients with severe ARDS requiring VV-ECMO.

Finally, fluid balance is compromised during intravenous treatment of Pneumocystis pneumonia in infants because of the large amount of solvent necessary to deliver cotrimoxazole. The drug is stable for $1 \mathrm{~h}$ at the maximum concentration (1/15 dilution; $[17,18])$. To deliver $100 \mathrm{mg} / \mathrm{kg} /$ day sulfamethoxazole and $20 \mathrm{mg} / \mathrm{kg} /$ day trimethoprim using the commercial preparation sulfamethoxazole $400 \mathrm{mg} / \mathrm{tri}$ methoprim $80 \mathrm{mg} / 5 \mathrm{~mL}$, the infant required about $20 \mathrm{~mL} / \mathrm{kg}$ solvent/day (i.e., $100 \mathrm{~mL}$ ). Fluid overload should be prevented by prescription of diuretics or via renal replacement therapy (RRT). Continuous RRT is feasible in children weighing up to $10 \mathrm{~kg}$ [19], but, in our case, it was easier and safer to link the ECMO and RRT circuits in parallel using the same cannula; this method is well-documented [20].

\section{Conclusion}

We describe here the first case of an infant with AIDS who developed refractory ARDS caused by Pneumocystis pneumonia and who was successfully treated via VV-ECMO. Improvement was slow, and severe extracorporeal circulation complications occurred during evolution, but decannulation with a favorable outcome, and ICU discharge, was possible after 30 days of VV-ECMO. AIDS per se should not be a contraindication for VV-ECMO, even in pediatric patients, although evolution will be longer in such patients. The fortuitous diagnosis of HIV infection indicates that 
systematic biological assessment of rare causes of refractory ARDS must be recommended for pediatric cases. Moreover, our case emphasizes the need to monitor the fluid balance during Pneumocystis pneumonia treatment because of the large amount of antibiotic-containing solvent delivered, creating a need for rapid VV-ECMO ultrafiltration to eliminate fluid overload.

\section{Abbreviations}

HIV: $\quad$ Human immunodeficiency virus

ARDS: $\quad$ Acute respiratory distress syndrome

ICU: Intensive care unit

VV- Venovenous extracorporeal membrane

ECMO: oxygenation

AIDS: $\quad$ Acquired immunodeficiency syndrome

PEEP: $\quad$ Positive end-expiratory pressure

ELSO: $\quad$ Extracorporeal Life Support Organization

PALICC: Pediatric Acute Lung Injury Consensus

Conference

RRT: $\quad$ Renal replacement therapy.

\section{Data Availability}

No data were used to support this study.

\section{Additional Points}

Summary. We emphasize that it is important to investigate rare causes of ARDS systematically and highlight the potential benefits of ECMO for such conditions.

\section{Disclosure}

The English in this document has been checked by at least two professional editors, both native speakers of English. For a certificate, please see: http://www.textcheck.com/ certificate/Zrqr3f.

\section{Conflicts of Interest}

The authors declare no conflicts of interest.

\section{Authors' Contributions}

Dr. Cane conceptualized the ideas of this case, collected data, and drafted the initial manuscript. Dr. Tafer and Prof. Ouattara designed and directed the project, conceptualized the ideas of the case, and reviewed and revised the manuscript. Drs. De Boislambert, Sgro, Lavedan, and Foulgoc reviewed and revised the manuscript. All authors approved the final manuscript as submitted and agree to be accountable for all aspects of the work.

\section{Acknowledgments}

The English language has been checked by at least two professional editors, both native speakers of English (for certificate, see http://www.textcheck.com/certificate/ Zrqr3f).

\section{References}

[1] J. J. Schmidt, C. Lueck, S. Ziesing et al., "Clinical course, treatment and outcome of Pneumocystis pneumonia in immunocompromised adults: a retrospective analysis over 17 years," Critical Care, vol. 22, no. 1, p. 307, 2018.

[2] A. T. Hernandez Conte, D. Ng, D. Ramzy et al., "Extracorporeal membrane oxygenation in a 29 -year-old man with Pneumocystis jirovecii respiratory failure and AIDS," Texas Heart Institute Journal, vol. 45, no. 4, pp. 254-259, 2018.

[3] N. Lee, D. Lawrence, B. Patel, and S. Ledot, "HIV-related Pneumocystis jirovecii pneumonia managed with caspofungin and veno-venous extracorporeal membrane oxygenation rescue therapy," BMJ Case Reports, vol. 2017, Article ID bcr2017-221214, 2017.

[4] H. S. Ali, I. F. Hassan, and S. George, "Extra corporeal membrane oxygenation to facilitate lung protective ventilation and prevent ventilator-induced lung injury in severe Pneumocystis pneumonia with pneumomediastinum: a case report and short literature review," BMC Pulmonary Medicine, vol. 16, no. 1, p. 52, 2016.

[5] F. G. De Rosa, V. Fanelli, S. Corcione et al., "Extra corporeal membrane oxygenation (ECMO) in three HIV-positive patients with acute respiratory distress syndrome," $B M C A n$ esthesiology, vol. 14, no. 1, p. 37, 2014.

[6] M. Penazzato, A. J. Prendergast, L. M. Muhe, D. Tindyebwa, and E. Abrams, "Optimisation of antiretroviral therapy in HIV-infected children under 3 years of age," Cochrane Database of Systematic Reviews, vol. 5, Article ID CD004772, 2014.

[7] A. J. Williams, T. Duong, L. M. McNally et al., "Pneumocystis carinii pneumonia and cytomegalovirus infection in children with vertically acquired HIV infection," AIDS, vol. 15, no. 3, pp. 335-339, 2001.

[8] ARDS Definition Task Force, "Acute respiratory distress syndrome: the Berlin definition," JAMA, vol. 307, no. 23, pp. 2526-2533, 2012.

[9] R. G. Khemani, L. S. Smith, J. J. Zimmerman, and S. Erickson, "Pediatric acute respiratory distress syndrome: definition, incidence, and epidemiology: proceedings from the pediatric acute lung injury consensus conference," Pediatric Critical Care Medicine, vol. 16, no. 1, pp. S23-S40, 2015.

[10] G. MacLaren, S. Conrad, and G. Peek, Indications for Pediatric Respiratory Extracorporeal Life Support, ELSO, Ann Arbor, MI, USA, 2015.

[11] M. B. Domico, D. A. Ridout, R. Bronicki et al., "The impact of mechanical ventilation time before initiation of extracorporeal life support on survival in pediatric respiratory failure: a review of the extracorporeal life support registry," Pediatric Critical Care Medicine, vol. 13, no. 1, pp. 16-21, 2012.

[12] M. Gupta, T. P. Shanley, and F. W. Moler, "Extracorporeal life support for severe respiratory failure in children with immune compromised conditions," Pediatric Critical Care Medicine, vol. 9, no. 4, pp. 380-385, 2008.

[13] A. Y. L. Lim, A. C. Mattke, J. E. Clark, A. Pinzon-Charry, N. Alphonso, and N. Kapur, "Acquired immunodeficiency from maternal chemotherapy and severe primary Pneumocystis infection in an infant," Case Reports in Pediatrics, vol. 2020, Article ID 5740304, 5 pages, 2020.

[14] V. Lindén, J. Karlén, M. Olsson et al., "Successful extracorporeal membrane oxygenation in four children with 
malignant disease and severe Pneumocystis carinii pneumonia," Medical and Pediatric Oncology, vol. 32, no. 1, pp. 25-31, 1999.

[15] H. Ehrén, K. Palmér, M. Eriksson, and B. Frenckner, "Pediatric ECMO for pulmonary support: experience from 12 cases," Acta Paediatrica, vol. 84, no. 4, pp. 442-446, 1995.

[16] B. Frenckner, "Extracorporeal membrane oxygenation: a breakthrough for respiratory failure," Journal of Internal Medicine, vol. 278, no. 6, pp. 586-598, 2015.

[17] P. F. Jarosinski, P. E. Kennedy, J. F. Gallelli, and W. G. Magnuson, "Stability of concentrated trimethoprimsulfamethoxazole admixtures," American Journal of HealthSystem Pharmacy, vol. 46, no. 4, pp. 732-737, 1989.

[18] K. W. Deans, J. R. Lang, and D. E. Smith, "Stability of trimethoprim-sulfamethoxazole injection in five infusion fluids," American Journal of Health-System Pharmacy, vol. 39, no. 10, pp. 1681-1684, 1982.

[19] J. M. Symons, P. D. Brophy, M. J. Gregory et al., "Continuous renal replacement therapy in children up to $10 \mathrm{~kg}$," American Journal of Kidney Diseases, vol. 41, no. 5, pp. 984-989, 2003.

[20] M. Ostermann, M. Connor, and K. Kashani, "Continuous renal replacement therapy during extracorporeal membrane oxygenation: why, when and how?" Current Opinion in Critical Care, vol. 24, no. 6, pp. 493-503, 2018. 